\title{
EPIDEMIOLOGICAL ANALYSIS ON 3614 PATIENTS WITH TEMPORO-MANDIBULAR DISORDERS (TMD) BASIC STATISTICAL ASPECTS
}

\author{
Jurkemik $\mathrm{J}^{1}$, Janickova $\mathbf{M}^{1}$, Malachovsky $\mathrm{I}^{1}$, Rozic $\mathrm{L}^{1}$, Chmurny $\mathbf{M}^{2}$, Statelova $\mathrm{D}^{1}$ \\ ${ }^{1}$ Clinic of Stomatology and Maxillofacial Surgery, ${ }^{2}$ Clinic of Orthopaedics, University Hospital in Martin, \\ Jessenius Faculty of Medicine in Martin, Comenius University in Bratislava, Slovakia
}

\begin{abstract}
The aim of this work was to present data from a large sample of patients with Temporo-Mandibular Disorders (TMD) in order to clarify some aspects of the development of pathological conditions that affect large parts of the population. In the past years there was a rapid growth of the incidence of the temporomandibular dissorders. The ethiopathogenesis is in most cases unclear. Based on the latest information supposed are the biopsychosocial factors.
\end{abstract}

Key words : temporo-mandibular disorders, statistical analysis, epidemiology

\section{INTRODUCTION}

The Temporo-Mandibular Disorders (TMD) are a set of dysfunctional patterns concerning the temporo-mandibular joints (TMJ) and the masticatory muscles with frequent involvement of other structures of various body districts with the result of making complicated the classification and the diagnostic processes (1). This complexity has led, in recent years, to consider the origin of the TMD as multifactorial both for the undoubted frequent association of several factors in causing the disease then for the current inability of the researchers to recognize the real factors causing the dysfunction $(2,3,4)$.

This preliminary work put in relation, through an epidemiological evaluation, anamnestic and clinical data collected from a sample of patients affected by TMD. An analysis of these data clearly showed that there were large quantities of variables involved in these disorders and which occur with a wide variety of possible clinical signs. This complexity, in accordance with the current knowledge that it is not able to clarify the etiology of these disorders, makes intricate both diagnostic then therapeutic aspects. The data founded in this report evaluate basic structural stastistics of the monitored group and MRI findings.

\section{MATERIAL AND METHODS}

The data analyzed in this work are the outcomes of the patients who spontaneously arrived requiring a specialist visit at the ambulance of temporomandibular joints disorders at the Department of Stomatology and Maxillofacial Surgery, Jessenius Faculty of Medicine in Martin. This specialized ambulance visited in the years 2007-2012 2179 patients and according to our retrospective study in the years 2010-2014 3614 patients. These data are showing a significant rise of patients diagnosed or treated with TMD in our department.

In this work the authors illustrated and described the epidemiological basic characteristics of the sample with the aid of elementary statistical techniques and tools.

\footnotetext{
A d d r e s s or corres pond e n c e:

Dagmar Statelová, MD, PhD., Assoc. prof., Clinic of Stomatology and Maxillofacial Surgery, Jessenius Faculty of Medicine and University Hospital in Martin, Kollarova Str. No 2, 03659 Martin, Slovakia; e-mail: Dagmar.Statelova@jfmed.uniba.sk
} 


\section{RESULTS}

The results showed that $83.6 \%$ of treated or diagnosed patients were women. The average age of the women patients was 35.8 years and of men it was 42.3 years. In the monitored group there is a significant rise of patients in the years 2010-2014 (Fig.1). According to the results the majority of the patients were sent to the Department of Stomatology and Maxillofacial Surgery, Jessenius Faculty of Medicine in Martin by the stomatologists $62.72 \%$. The othorhinolaryngologists have diagnosed temporomandibular arthropathy in $24.36 \%$ of the patients, general practinioner in $10.23 \%$, neurologist in $2.87 \%$ (Fig.2).

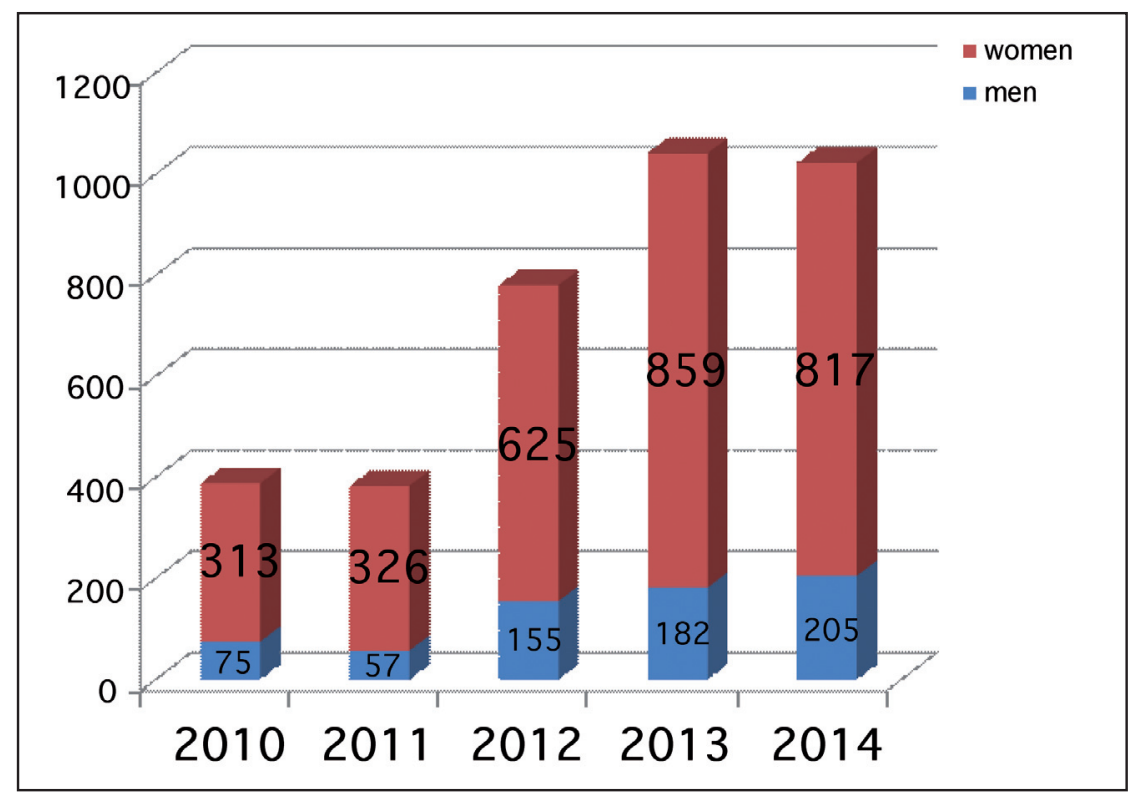

Fig.1 The sex relation of treated or diagnosed patients

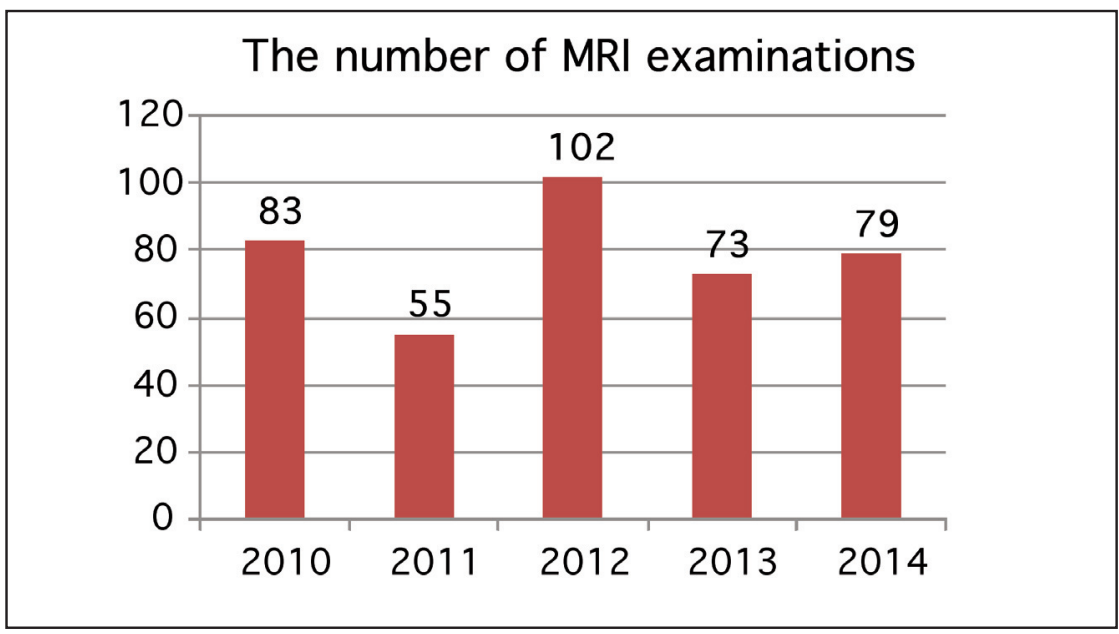

Fig.2 The number of MRI examinations of the TMJ 
Patients with severe clinical picture of arthropathy or patients resistent to the conservative therapy have been sent to MRI examination. From the total number of patients 3614 treated or diagnosed in the years 2010-2014 10.85\% (392) of the patients underwent MRI examination of the temporomandibular joint (Fig. 2). The MRI was repeated in all cases after one year of therapy. The results in table. 3 are evaluation of all MRI examinations and findings on each single TMJ. The most frequent grade of internal derangement was the second with the score $25.92 \%$ folowed by the first grade $-21.64 \%$, the third grade $11.25 \%$, the fourth grade $1.47 \%$. Arthrosis and damage of the cartilage was diagnosed in $16.50 \%$ of cases. Without pathology were $23.23 \%$ of the examined TMJs.

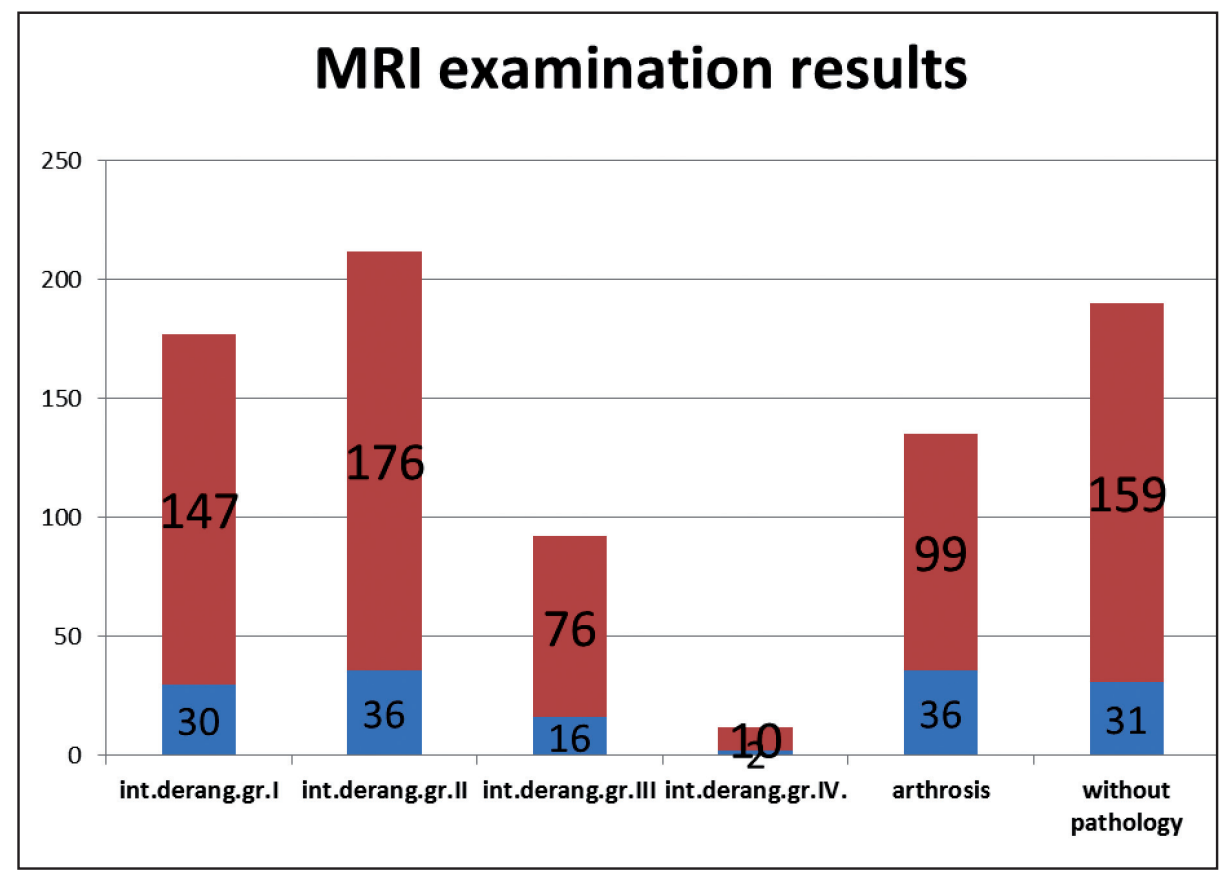

Fig. 3 MRI examination results

Depending on the results of MRI the arthroscopy of the temporomandibular joint was indicated in 73 cases from monitored group of 392 patients. 76\% of this monitored group were women, $24 \%$ were men, the average age was 35.6 years, what is in correlation with the large cohort patients group treated or diagnosed in years 2010-2014 where the average age was 35.8 years. From the 73 endoscopically examinated temporomandibular joints in $46 \%$ there was MRI verified internal derangement gradus III., in 35\% gradus II., in 14\% gradus IV. and in 6\% gradus I. In 48\% the arthroscopy was performed on the right TMJ, in 39.7\% it was performed on the left TMJ and in $12.3 \%$ bilaterall. For the 73 arhtroscopically treated patients who had finished their course of treatment and had also completed a period of post-treatment control it has been evaluated that the average range of abduction has raised from $31 \mathrm{~mm}$ on the beginning of the treatment to $42 \mathrm{~mm} 12$ months after the arthroscopy of the TMJ.

According to the results the muscular pain was present in the $23.7 \%$ of the cases. The parafunction bruxism was highlighted in $8.6 \%$ of cases. Clenching of the teeth has been studied by other authors to investigate its prevalency and relationship to the TMD. According to the epidemiological study published by Di Paolo in 2013 the clenching was positive in $28.5 \%$ of subjects analyzed; such last parafunction seems to be the most widespread and the most potentially harmful for the individuals who are affected (1). ( Fig.4). 


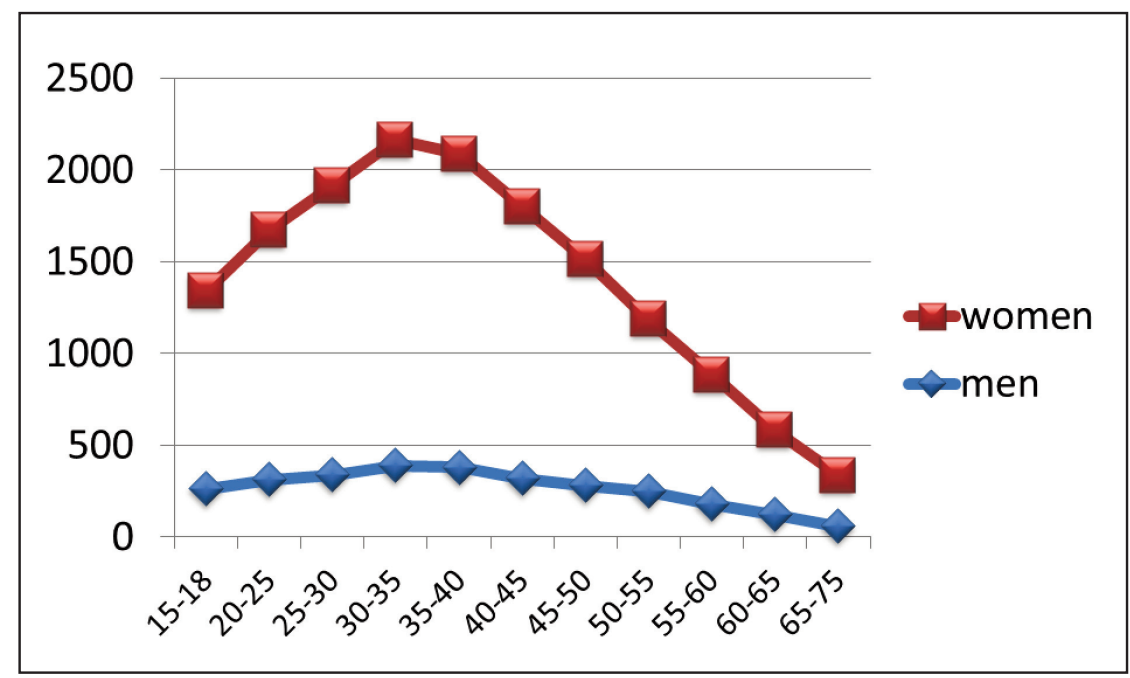

Fig.4 Age correlation between men and women in the monitored group

We observed that in 33.7\% of asymptomatic patients after MRI examination there was disc dislocation and also according to the results in $23.23 \%$ of MRI examinated joints were no pathology while the clinical picture supported damage of intraarticular structures.

\section{DISCUSSION}

The data collected in this preliminary retrospective study were subjected to a simple statistical analysis to describe the basic epidemiology aspects of a large sample of patients with TMJ dysfunctions.

The term bruxism is defined as a repetitive jaw muscle activity characterized by clenching or grinding of the teeth and/or by bracing or thrusting of the mandible (5). Currently, bruxism is no longer accepted as a single entity, but is divided into two distinct entities - awake and sleep bruxism - based on when the activity occurs. An excessive loading of the hard and soft tissues of the joint is suggested to be the cause of joint disorders. Thus, prolonged clenching can cause degenerative changes due to the overloading induced with time. Damage to the articular disc can be the cause of TMD. Some researchers have shown that acute mechanical overloads in vivo can cause severe cartilage damage $(6,7)$. Bruxism is considered as the most severe parafunctional activity in relationship to the temporomandibular disorders although there are some issues concerning the causal relationship to ethipathogenesis of the TMD (8). In this study the investigations of bruxism were based on self-report or clinical bruxism. This may be the reason of a low incidency of bruxism in our study. Sleep bruxism is no longer considered to be simply related to mechanistic factors, such as occlusal discrepancies, or a result of psychological issues, such as stress, anxiety or depression or a combination thereof $(9,10)$. Instead, most authorities now consider sleep bruxism to be primarily a sleep-related movement disorder with a yet to be discerned multifactorial etiology and complex multisystem physiological processes.

According to the results MRI examination was performed in $10.85 \%$ of the patients. These patients were either resistant to the conservative treatment of the TMJ and without severe clinical pathology due of which MRI was not indicated at the beginning of the treatment or with a severe clinical pathology due to which MRI examination was necessary for objectification of the intraarticular pathology in addition to clinical examinations. 


\section{CONCLUSIONS}

Studies have indicated that the prevalence of bruxism is highest between adolescence and 49 years, and that it decreases with age $(11,12,13)$. It is difficult to differentiate the effects of bruxism on the soft and hard tissues of the stomatognathic system, because many individuals are unaware of their parafunctional habits. In this study there was a significant correlation between bruxism and internal derangement of the TMJ. The role of MRI, a reliable, non-invasive procedure, in the diagnosis of internal derangements of the TMJ is well described. MRI is best suited to demonstrating disc abnormalities and macroscopic bony changes. MRI provides adequate morphological bony detail and does not need to be routinely complemented by CT or conventional tomography. Degenerative bony changes and the relationship between the disc and condyle were clearly seen on MR images. Our study showed that clinically discernible sounds tend to reveal a change in the relationship between the condyle and the disc at the degree of jaw separation where the sound occurs. Joint sounds might be caused by a variety of pathological processes such as disc displacement, condylar subluxation, deviations in the form or shape of articulating surfaces, and the presence of fibrous bands or adhesions within the joint. Bruxism, especially clenching and grinding, may be indirectly related to TMJ sounds. Joint sounds reveal information about the relationship between the disc and condyle and crepitation which might develop with bruxism.

The direction of parafunctional forces in grinding is horizontal and is not tolerated because eccentric mandibular movements are involved. Bruxism, especially clenching, could result in an internal derangement of the TMJ. It is generally believed that pathological destruction of articular tissue occurs when articular remodelling does not maintain a balance between its structure and function. The results of this study confirmed the high prevalence of bony changes in bruxing patients.

In conclusion, this study demonstrated that a high prevalence of condylar bony changes occurred in reducing joints in patients with bruxing behaviour. We also believe that the results presented here are useful as control data when considering the treatment of internal derangement of the TMJ. Further research is needed to understand the influence of the duration of diurnal and nocturnal bruxism on the internal derangement of the TMJ. Also the prevalence of internal derangement as a result of bruxism and clenching is highest in the age groups 20-40 years which can be considered as an active lifestyle in people who suffer under stress everyday life conditions. All of this can result in psycho-emotionall pathological chewing muscle synergism.

\section{REFERENCES}

1. Di Paolo C, Cascone P. Patologia dell'articolazione Temporomandibolare. UTET. 2004.

2. Newman Graff-Radford. The role of temporomandibolar disorders and cervical dysfunction in tension-type headache. Curr Pain Headache Rep. 2002 Oct.

3. Magnusson T, Syren M. Therapeutic jaw exercises and interocclusal appliance therapy. A comparison between two common treatments of temporomandibolar disorders. Swed Dent. 1999 Jan.

4. Di Paolo C, Costanzo GD, Panti F, et al. Epidemiological analysis on 2375 patients with TMJ disorders: basic statistical aspects. Annali di Stomatologia. 2013;4(1):161-169. doi:10.11138/ads.0161.

5. Lobbezoo F, Ahlberg J, Glaros AG et al. Bruxism defined and graded: an international consensus. J Oral Rehabil 2013; 40(1): 2-4.

6. Radin EL, Martin RB, Burr DB et al. Effects of mechanical loading on the tissues of the rabbit knee. J Orthop Res 1984; 2(3): 221-234.

7. Thompson RC, Oegema TR, Lewis JL et al. Osteoarthrotic changes after acute transarticular load: an animal model. J Bone Joint Surg 1991; 73(7): 990-1001. 
8. Manfredini D, Lobbezoo F. Relationship between bruxism and temporomandibular disorders: a sys tematic review of literature from 1998 to 2008. Oral Surg Oral Med Oral Pathol Oral Radiol Endod 2010; 109(6): e26-e50.

9. Ramfjord SP. Bruxism, a clinical and electromyographic study. J Am Dent Assoc. 1961;62:21-44.

11. Manfredini D, Lobbezoo F. Role of psychosocial factors in the etiology of bruxism. J Orofac Pain 2009;23(2):153-66.

10. Hartmann E, Mehta N, Forgione A. Bruxism: effects of alcohol. Sleep Res 1987; 16: 351-353.

12. Spruijt RJ, Wabeke KB. Psychological factors related to the prevalence of temporomandibular joint sounds. J Oral Rehabil 1995; 22: 803-808. CrossRef, Medline

13. Westling L, Carlsson GE, Helkimo M. Background factors in craniomandibular disorders with special reference to general joint hypermobility, parafunction, and trauma. J Craniomandib Disord 1990; 4: 89-98.

Received: September,7,2015

Accepted: December,10,2015 